\title{
Evaluation of culture media for the production of secondary metabolites in a natural products screening program
}

\author{
Karen M VanderMolen, Huzefa A Raja, Tamam El-Elimat and Nicholas H Oberlies*
}

\begin{abstract}
Variation in the growing environment can have significant impacts on the quantity and diversity of fungal secondary metabolites. In the industrial setting, optimization of growing conditions can lead to significantly increased production of a compound of interest. Such optimization becomes challenging in a drug-discovery screening situation, as the ideal conditions for one organism may induce poor metabolic diversity for a different organism. Here, the impact of different media types, including six liquid media and five solid media, on the secondary metabolite production of three fungal strains was examined in the context of the drug-discovery screening process. The relative production of marker compounds was used to evaluate the usefulness and reliability of each medium for the purpose of producing secondary metabolites.
\end{abstract}

Keywords: Media; Screening; Secondary metabolites; Fungi

\section{Introduction}

Fungi provide a plentiful and diverse source of unique and often bioactive metabolites, and they have produced a number of medicinally important compounds, including penicillin, mevinolin (Lovastatin) (Gloer, 2007), fingolimod (Strader et al., 2011), and caspofungin (Keating \& Figgitt, 2003). The search for new and active compounds from microbial sources is a pursuit for many natural products laboratories. Typically, these efforts will employ a standard culture procedure that most or all microbial strains pass through as a preliminary step to the natural products discovery process. The manipulation of environment and nutrition has been shown to have substantial impacts on the quantity and diversity of secondary metabolite production (Fiedurek et al., 1996; Bode et al., 2002; Miao et al., 2006; Bills et al., 2008; Xu et al., 2008; Mohanty \& Prakash, 2009; Kossuga et al., 2012; Shang et al., 2012), and optimization of this initial step, the microbial culture, may have profound effects on the output and success of a natural products screening program.

\footnotetext{
*Correspondence: nicholas_oberlies@uncg.edu

Department of Chemistry and Biochemistry, University of North Carolina at
} Greensboro, Greensboro, NC, USA

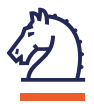

Methodical modification of culture growing conditions is often referred to as the OSMAC (one strain, many compounds) approach (Bode et al., 2002; Scherlach \& Hertweck, 2006). This concept is ideal for fully exploiting the metabolomic diversity of a single or handful of organisms, but it becomes cumbersome when applied to a large-scale screening program. Applying a suite of culture conditions for even one factor, whether it is media, salinity, $\mathrm{pH}$, temperature, etc., to every screened organism quickly becomes so labor and material intensive as to be impractical. The aim of this study, therefore, was to determine an ideal culture medium that could be applied to a drug discovery screening program as a standard preliminary medium.

This ideal medium would have to fit several criteria. In a medium-throughput but academic setting, large volumes of media will be used, so only inexpensive, readily available, and easily prepared media were evaluated. In addition, some media that have shown promise as metabolite inducers in other studies, such as vermiculite (Bills et al., 2012), were excluded due to the high startup cost necessary for such cultures. Our aim was to evaluate different media that could be integrated into the screening process with minimal changes to the existing equipment available. Six liquid broths and five solid- 
state media were chosen for evaluation. Three fungal strains were grown on each medium, and the resulting metabolite profiles were assessed.

In addition to a qualitative examination of the metabolite profile, the production of several marker compounds was tracked. Each fungus chosen for this study was previously studied and characterized by our laboratory, and each produced a known compound sharing many structural features with compounds that our research group isolates. Two [Glomerella acutata (G2) and a Hypocreales sp. (G24)] were isolated as endophytes from pawpaw (Asimina triloba L., Annonaceae) leaves collected in North America. The third strain, a Fusicolla sp. (G142), was isolated from freshwater submerged wood collected in North America. All strains used belonged to Hypocreales, of the phylum Ascomycota (Kirk et al., 2011). G2 and G142 both produced aurofusarin, while G24 produced PC3. The goal was to identify two distinct fungi, one an endophyte and one from freshwater, that produced identical secondary metabolites, and two from the same source, endophytes from pawpaw, that biosynthesized distinct metabolites. The production of these metabolites was tracked to provide a more in-depth assessment of the fungal extracts.

\section{Materials and methods Isolation of fungi}

Study site, collection and isolation of fungal endophyte strains. Healthy living twigs and leaves of pawpaw (Asimina triloba L., Annonaceae) were collected at random from a mature tree located on a private property in Indianapolis, Indiana, USA in July 2011 (9012 Colgate St.). Extra moisture was removed from the samples, and they were transported back to the laboratory in paper bags, where they were processed within 24-48 hours (Stone et al., 2004). In the lab, the twigs and leaves were cut into small pieces (approximately $2-5 \mathrm{~mm}$ in length) and washed in distilled water $\left(\mathrm{H}_{2} \mathrm{O}\right)$. Subsequently, the segments were surface-sterilized by sequential immersion in 95\% ethanol (EtOH; $10 \mathrm{~s})$, sodium hypochlorite (10-15\% available chlorine; $2 \mathrm{~min}$ ), and $70 \% \mathrm{EtOH}$ (2 min) (Stone et al., 2004). After the sterilization procedure, the plant segments were washed in distilled $\mathrm{H}_{2} \mathrm{O}$ and allowed to dry. The surface-sterilized segments were then transferred using sterile conditions onto $2 \%$ malt extract agar [MEA; Difco, $20 \mathrm{~g}$ MEA, $1 \mathrm{~L}$ sterile distilled $\mathrm{H}_{2} \mathrm{O}$ amended with streptomycin sulfate $(250 \mathrm{mg} / \mathrm{L})$ and penicillin $G(250 \mathrm{mg} / \mathrm{L})]$. To test the efficiency of the surface-sterilization procedure, and to confirm that emergent fungal colonies were indeed endophytic and not of epiphytic origin, individual surface-sterilized leaf and stem segments were spread on separate $2 \%$ MEA plates with antibiotics; the absence of fungal growth on the nutrient media confirmed the effectiveness of the sterilization procedure (Schulz et al., 1993). Plates were sealed with parafilm and incubated at room temperate in $12 \mathrm{~h}$ dark/light cycles until the emergence of fungal colonies was observed. Axenic cultures of emergent fungal endophytes are maintained at the University of North Carolina at Greensboro, Department of Chemistry and Biochemistry Fungal Collection and have been deposited as described below.

Isolation of fungi from submerged wood in fresh water. Submerged, dead woody debris was collected randomly in a freshwater lake in North Carolina, USA in October 2011 (Lake Brandt, 36 $10^{\prime} 1^{\prime \prime} \mathrm{N}, 1^{\circ} 50^{\prime} 18^{\prime \prime} \mathrm{W}$ ). Efforts were made to identify and collect substrates that had been submerged for a considerable time. This was estimated by observation of the degree of softening by fungal soft rot and colonization by other aquatic organisms. Samples were further processed in the laboratory to obtain axenic fungal cultures using established procedures (Shearer et al., 2004; Raja et al., 2009). Axenic cultures of freshwater fungi are maintained at the University of North Carolina at Greensboro, Department of Chemistry and Biochemistry Fungal collection and have been deposited as described below.

Molecular identification of fungal strains by DNA extraction, PCR and sequencing. Methods employed to identify fungi isolated in this study using the nuclear ribosomal internal transcribed spacer (ITS) region 1, 2, along with the short structural gene (5.8S) have been outlined in the supporting information of earlier studies (El-Elimat et al., 2013b; El-Elimat et al., 2013c; Figueroa et al., 2013). The ITS region was chosen to identify fungal species, as it has been recently identified as a barcode marker for fungi (Schoch et al., 2012). Sequence data were deposited in GenBank; the accession numbers for G2, G24, and G142 were AB858344, AB858345, and AB858346, respectively. Cultures were deposited with the Leibniz Institute DSMZ-German Collection of Microorganisms and Cell Cultures; the accession numbers for G2, G24, and G142 were DSM 27861, DSM 27862, and DSM 27863, respectively.

\section{Liquid media cultivation}

Several liquid media were chosen to represent a broad range of nutrient sources, including both defined and undefined broths. Each fungal strain was first cultivated on $2 \%$ malt extract agar. Plugs from these cultures were used to inoculate $50 \mathrm{~mL}$ of six different culture broths in $250 \mathrm{~mL}$ Erlenmeyer flasks. These broths included Czapek Dox (CD; Sigma Aldrich), 2\% Malt Extract (ME; Difco), Potato Dextrose (PD; Difco), YPSS (4 g yeast extract, $14 \mathrm{~g}$ soluble starch, $1 \mathrm{~g}$ dibasic $\mathrm{K}_{2} \mathrm{HPO}_{4}, 0.5 \mathrm{~g}$ $\mathrm{MgSO}_{4} \cdot 7 \mathrm{H}_{2} \mathrm{O}, 1 \mathrm{~L} \mathrm{H}_{2} \mathrm{O}$ ), YESD (20 g soy peptone, $20 \mathrm{~g}$ dextrose, $5 \mathrm{~g}$ yeast extract, $1 \mathrm{~L} \mathrm{H}_{2} \mathrm{O}$ ), and PYG (1.25 g soy peptone, 1.25 g yeast extract, $5 \mathrm{~g}$ D-glucose, $1 \mathrm{~L} \mathrm{H}_{2} \mathrm{O}$ ). 
Liquid cultures were grown for 14 days on an orbital shaker $(100 \mathrm{rpm})$ at room temperature before extraction. Each culture was grown in triplicate.

\section{Solid media cultivation}

Five forms of solid media, including rice, grits, oatmeal, wheat germ, and a 3:1:1:1 mixture of the same were used. For the preparation of solid media, $10 \mathrm{~g}$ of medium were mixed with $25 \mathrm{~mL}$ of deionized $\mathrm{H}_{2} \mathrm{O}$ in a $250 \mathrm{~mL}$ Erlenmeyer flask, sealed with a foam plug and tinfoil, and autoclaved. Plugs (from the $2 \%$ malt extract agar cultures listed above) of each fungus were used to inoculate $15 \mathrm{~mL}$ of YESD. These seed cultures were grown for three days on an orbital shaker (100 rpm) at room temperature, and these were used to inoculate the solid media, which was allowed to grow (stationary) for an additional 11 days at room temperature. Each culture was grown in triplicate.

\section{Extraction of fungal cultures}

To each culture, $60 \mathrm{~mL}$ of 1:1 chloroform:methanol $\left(\mathrm{CHCl}_{3}: \mathrm{MeOH}\right)$ were added. Solid media cultures were chopped with a spatula to ensure proper mixing. The cultures were shaken overnight $(100 \mathrm{rpm})$ at room temperature and subsequently filtered by vacuum. The filtrate was stirred for $1 \mathrm{~h}$ with $90 \mathrm{~mL}$ of $\mathrm{CHCl}_{3}$ and $100 \mathrm{~mL}$ deionized $\mathrm{H}_{2} \mathrm{O}$. This mixture was then transferred to a separatory funnel, and the bottom organic layer was evaporated to dryness in vacuo. The dried or-

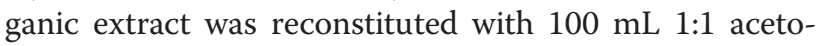
nitrile $\left(\mathrm{CH}_{3} \mathrm{CN}\right): \mathrm{MeOH}$ and $100 \mathrm{~mL}$ hexanes. This biphasic solution was stirred for $1 \mathrm{~h}$ and transferred to a separatory funnel. The bottom, defatted organic extract was evaporated to dryness in vacuo.

\section{Analysis of fungal metabolite profiles by ultra performance liquid chromatography-mass spectrometry (UPLC-MS)}

The defatted organic extract of each fungal culture was examined by UPLC-MS. The method used was developed as part of a dereplication procedure (El-Elimat et al., 2013a). In brief, analyses were performed using a Waters Acquity UPLC system utilizing a BEH C18 column (Waters; $50 \mathrm{~mm} \times 2.1 \mathrm{~mm}$ i.d., $1.8 \mu \mathrm{m}$ ). The mobile phase consisted of $\mathrm{CH}_{3} \mathrm{CN}$ and $0.1 \%$ formic acid- $\mathrm{H}_{2} \mathrm{O}$, increasing linearly from $15 \% \mathrm{CH}_{3} \mathrm{CN}$ at the time of injection to $100 \% \mathrm{CH}_{3} \mathrm{CN}$ at $10 \mathrm{~min}$. The flow rate was $0.3 \mathrm{~mL} / \mathrm{min}$ and column temperature was $40^{\circ} \mathrm{C}$. The UPLC system was coupled to a Thermo Scientific LTQ Orbitrap XL equipped with electrospray ionization (ESI) source. The production of several known metabolites (Figure 1) was tracked using high-resolution mass spectrometry (HRMS). Crude extracts were dissolved in 1:1 $\mathrm{MeOH}$ :dioxane at a concentration of $2.0 \mathrm{mg} / \mathrm{mL} ; 6.0 \mu \mathrm{L}$ were injected, and the peak areas of each metabolite

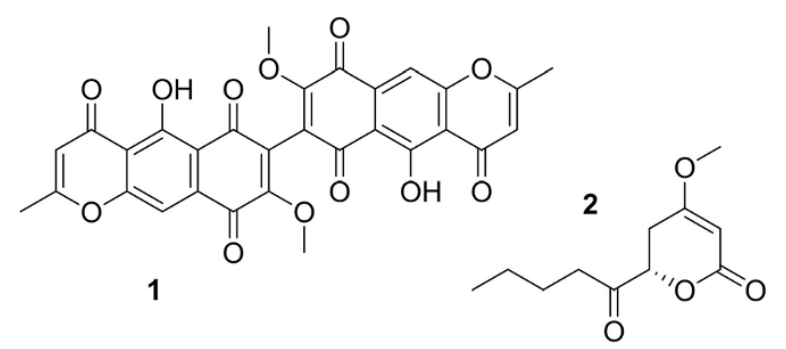

Figure 1 Marker compounds used to track the production potential of various media; (1) aurofusarin, (2) PC3.

measured. Additionally, extracts were also examined using the same UPLC system equipped with an evaporative light scattering detector (ELSD; Waters); all other conditions were the same.

\section{Results}

\section{Effects of media on fungal growths}

The visual differences in fungal growth were the most immediately apparent result of varying the culture medium and were a primary instigator of this study; striking differences in color occurred as various secondary metabolites were stimulated by a new medium (Figure 2). These color changes were most notable in liquid media, but the amount of sporulation (orange) and exudate (black) produced varied between solid media, too. As an example, after the 11 days of growth on solid media, the rice cultures of G2 exhibited almost no sporulation or exudate (Figure 2), while the grits culture showed a small amount of black exudate, the oatmeal culture exhibited heavy sporulation (orange), and the wheat germ culture had a large amount of both.

\section{Effects of media on the profile of secondary metabolites} The various media tested exhibited marked differences in the mass of extract (Figure $3 \mathrm{a}$ and b). On the whole, the cultures grown on solid media produced extracts with masses one to two orders of magnitude larger than the same fungus grown in any of the liquid media. In both solid and liquid cultures, G2 consistently produced the lowest mass of extract, though this difference was more notable in the liquid media. The variation of the amount of extract produced was greater in liquid media than solid. The greatest variation between liquid media was shown by G24; in Czapek Dox broth, the culture produced less than two mg of total defatted extract, while it produced more than $20 \mathrm{mg}$ when grown in potato dextrose broth and YESD broth (Figure 3a).

The mass of the defatted extract was also compared to the mass of the extract prior to the defatting step of the extraction procedure (Figure $3 \mathrm{c}$ and $\mathrm{d}$ ). Defatting is an important step in the extraction process for two reasons. The hexane wash eliminates some common but unwanted 


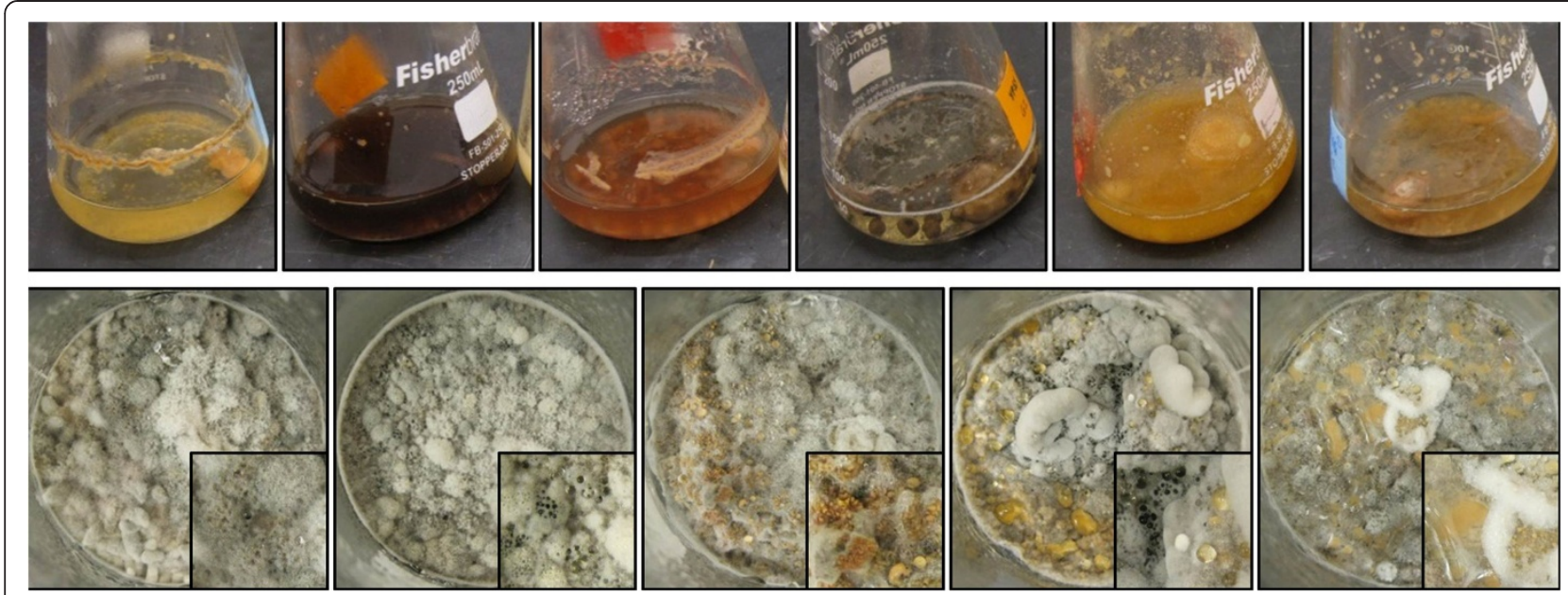

Figure 2 Photos of G2 in liquid media (top); from left to right: Czapek dox, 2\% malt extract, potato dextrose, YPSS, YESD, and PYG broths. Photos of G2 on solid media (bottom); from left to right: rice, grits, oatmeal, wheat germ, and 3:1:1:1, respectively, of the same. Enlargements of the photos $(2.5 \times)$ are provided in the lower right hand corner to help visualize the production of spores (orange) and/or exudates (black).

metabolites, such as fatty acids, that are not of interest to most natural product screening programs. It also cleans the extract of the very nonpolar constituents that are detrimental to reverse phase columns. Analyzing the percentage of extract lost in this defatting step can help to characterize the composition of the fungal extract.
Extracts from solid media cultures retained a much smaller percentage of the original mass, typically between 10 and 30\%, though G142 retained higher percentages of 42 and $52 \%$ on grits and the 3:1:1:1 mixture, respectively. Growths on liquid media, on the other hand, retained between 70 and $90 \%$ of their mass after the defatting wash,
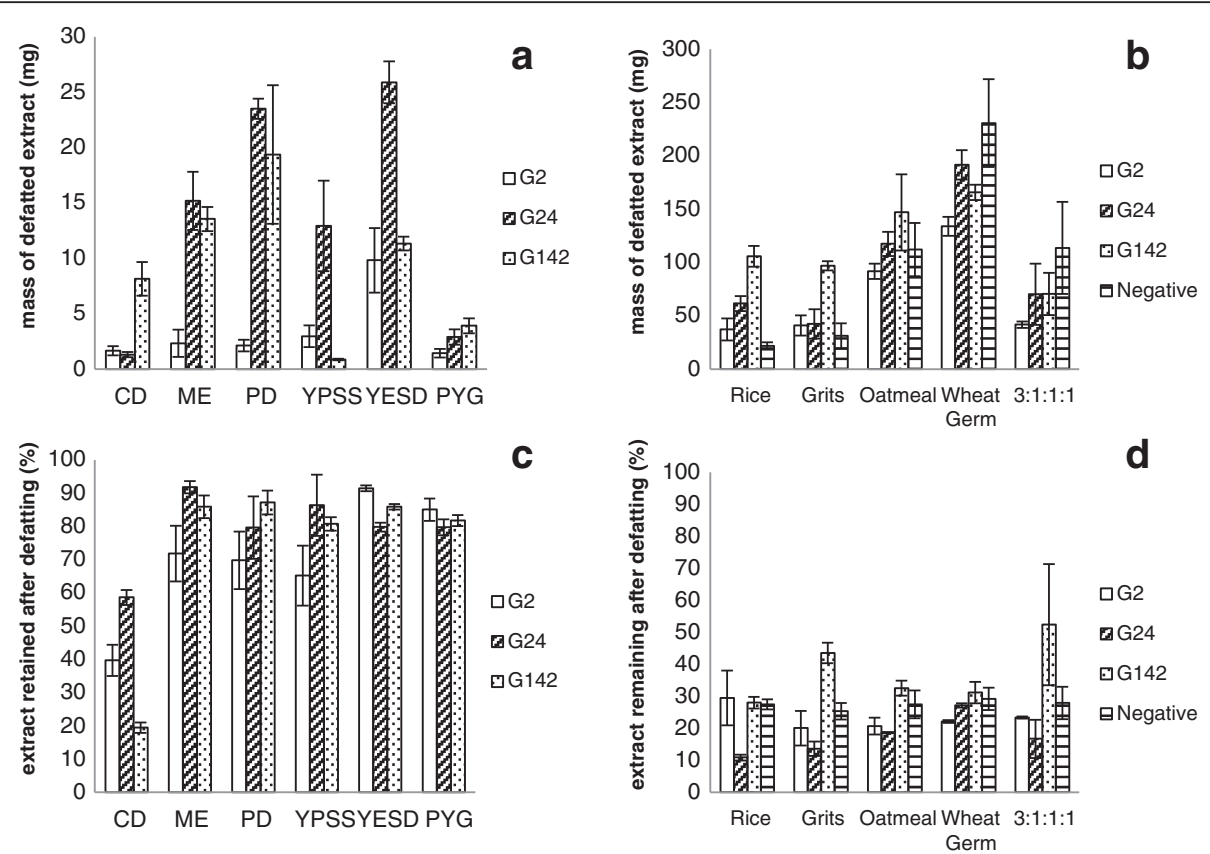

Figure 3 A comparison of the (a) mass of defatted extract of fungal growths in liquid media, (b) mass of defatted extract of fungal growths on solid media, (c) percentage of organic extract retained after the defatting step (liquid media), and (d) the percentage of organic extract retained after the defatting step (solid media). Data plotted are means \pm SD of three replicate growths per culture medium. The "Negative" series in solid media ( $b$ and $d$ ) refers to the mass of extracted solid media alone, with no fungal growth. The same data for liquid cultures were negligible and therefore not shown. Abbreviations of media are defined in the Methods section. 


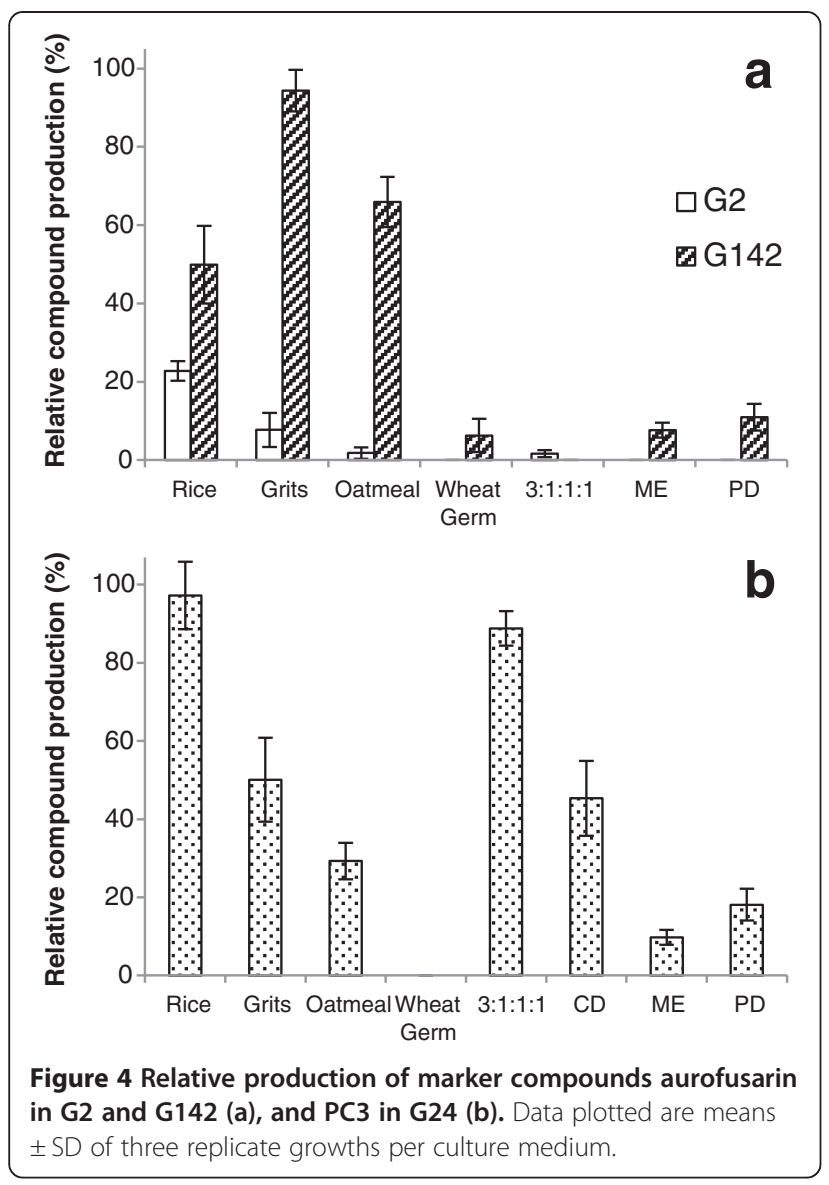

though all three fungi grown on Czapek Dox lost significant portions of their extract in this step.

\section{Effects of media on composition of extract}

The UPLC profiles of the fungal extracts showed wide variation between media (Additional file 1). Particularly in the liquid cultures, metabolites with vastly different retention times were produced. Grown in $2 \%$ malt extract broth (ME), G142 produced nine major peaks that eluted between 4 and $6.5 \mathrm{~min}$ on a $10-\min \mathrm{CH}_{3} \mathrm{CN}$ gradient. The same fungus grown in YPSS produced only five major peaks that all eluted after 7.5 min (Additional file 1).

The production of marker compounds (Figure 1) was compared between media by measuring the peak areas of each metabolite via UPLC-MS and multiplying by total mass of the defatted extract, in order to more accurately compare the total production of each metabolite instead of the percentage of extract the metabolite comprised. This value was expressed as relative percentage of metabolite production, normalized to the largest value of each metabolite's set. Aurofusarin was the metabolite of interest in both G2 and G142, while the metabolite of interest produced by G24 was PC3. Aurofusarin is a mycotoxin and red pigment common in Fusarium species (Ezekiel et al., 2012; Soerensen et al., 2012; Mikusova et al., 2013). PC3 is an analog of pestalotin, which was first described as a product of an unidentified fungus in 1973 (Strunz et al., 1974); pestalotins synergistically augment the plant hormone gibberellin (Kimura et al., 1971; Kimura et al., 1977; Kirihata et al., 1996). While the structural diversity of compounds from fungi can be vast (El-Elimat et al., 2012), these were chosen as representative compounds for a drug screening program, since they contain heteroatoms, conjugated rings, carbonyl groups, hydroxy, methoxy, and methyl moieties, and polyketide derived chains.

For all fungi, rice and grits consistently produced greater quantities of the marker compounds, while wheat germ consistently produced little or no marker compound. Few of the liquid cultures produced meaningful quantities of the marker compounds (Figure 4), though they were detectable in most (Table 1). G2 produced modest amounts of aurofusarin when grown on oatmeal, when compared to the production when grown on rice; however, G142 consistently produced much greater quantities of aurofusarin on rice, grits, and oatmeal. While G2 produced little or no aurofusarin in the liquid cultures, G142 produced quantities in ME and PD comparable to the production by $\mathrm{G} 2$ on several solid cultures (Figure 4). PC3 production was highest when G24 was grown on rice and the 3:1:1:1 mixture, though its production in $\mathrm{CD}, \mathrm{ME}$, and $\mathrm{PD}$ were similar to the production on solid media.

\section{Discussion}

It has been shown that media conditions have varying effects on the production of fungal secondary metabolites (Bode et al., 2002; Miao et al., 2006). Often, the variation of culture conditions is used to optimize the yields of a specific compound, such as the active metabolite in a medicinal fungus (Xu et al., 2008) or drugproducing microbe (Pu et al., 2013). In other cases, the

Table 1 Summary of the production of marker compounds in solid and liquid media

\begin{tabular}{|c|c|c|c|c|c|c|c|c|c|c|c|}
\hline & Rice & Grits & Oatmeal & Wheat germ & $3: 1: 1: 1$ & CD & ME & PD & YPSS & YESD & PYG \\
\hline Aurofusarin (G2) & $\mathrm{m}$ & $\mathrm{m}$ & $\mathrm{m}$ & $d$ & $\mathrm{~m}$ & u & $d$ & $d$ & $d$ & u & u \\
\hline PC3 (G24) & $\mathrm{m}$ & $\mathrm{m}$ & $\mathrm{m}$ & d & $\mathrm{m}$ & $\mathrm{m}$ & $\mathrm{m}$ & $\mathrm{m}$ & $d$ & u & u \\
\hline Aurofusarin (G142) & $\mathrm{m}$ & $\mathrm{m}$ & $\mathrm{m}$ & $\mathrm{m}$ & $d$ & $d$ & $\mathrm{~m}$ & $\mathrm{~m}$ & $d$ & $d$ & $\mathrm{~m}$ \\
\hline
\end{tabular}

Table 1 Detection of marker compounds in solid and liquid media extracts; $m=$ measured, as shown in bar graphs (Figure 4), $d=$ detected at low levels, $\mathrm{u}=$ undetected. Limit of detection $0.22 \mathrm{mg} / \mathrm{g}$ extract. 
OSMAC approach can be used to fully exploit the biodiversity of a small number of microbes, usually fewer than five (Hestbjerg et al., 2002; Paranagama et al., 2007; Shang et al., 2012). These techniques can be very valuable for manipulating the metabolomes of several fungi at a time, but they also increase the manpower necessary to process a single culture by several fold for each growth condition being manipulated. Our laboratory processes approximately 400 new fungal cultures every year; applying the OSMAC approach to each and every culture would be impractical without the implementation of specialized equipment and procedures for the parallel cultivation of screened fungi (Bills et al., 2008).

This study was designed to evaluate media with the intention of optimizing a drug-discovery screening program, which is a different goal than finding the ideal medium for a specific microbial strain producing one or two key metabolites. What may be the best medium for one fungus could often prove unproductive for other strains. For example, G2 produced very little aurofusarin when grown on oatmeal, while G142 produced quantities equivalent to its production on rice. In potato dextrose broth, G24 and G142 produced large extracts, but the extract of G2 was less than $2 \mathrm{mg}$. Similarly, media that facilitate increased fungal growth and large amounts of extract may not produce compounds of interest. G24, when grown in PD or YESD liquid broths, produced extracts of similar masses to both each other and to its extracts when grown on rice, yet the metabolite of interest (PC3), was produced in significantly smaller quantities in PD and undetected in YESD. Thus, media in this study were evaluated with respect to both the amount of extract produced and the composition of that extract, including the production of the chosen marker compounds (Figure 1). Because the media were being assessed for use in a screening program, consistency of production across all three fungal strains was a more important criterion than excellent production in just one of the strains.

The variation in extract composition and marker compound production was more pronounced than expected when this study was designed. The most surprising outcome was that the marker compounds were undetected by UPLC-MS in several of the liquid culture extracts (Table 1). This was surprising, given that the limit of detection for the method was approximately $0.22 \mathrm{mg} / \mathrm{g}$ extract (El-Elimat et al., 2013a). Indeed, the amount of extract produced was often so drastically smaller than those produced by solid media cultures, that for a drugdiscovery screening program, it would be impractical to use liquid cultures as a standard culture procedure; isolating sufficient quantities of compounds of interest would be challenging. While it was enticing that the liquid cultures produced a lower percentage of fats than the solid media cultures, since fats complicate the purification process (Figure 3c and d), the overall paucity of metabolites negated this benefit.

The production of marker compounds varied widely between all cultures. On oatmeal, G142 (isolated from freshwater) produced amounts of aurofusarin similar to cultures grown on rice; G2 (an endophyte) had very poor production on oatmeal. The use of only G142 in such a media survey would indicate that oatmeal may be an ideal candidate as a standard solid medium, while the added information provided by using the second fungus producing the same compound refuted this conclusion. A further example was the production of PC3 by G24 when grown on the 3:1:1:1 mixture. Again, while PC3 was produced in quantities similar to that of the rice or grits media, G2 and G142 showed poor aurofusarin production on this same medium. In general, rice appeared to more consistently produce high quantities of the metabolites of interest, while wheat germ consistently did not.

For the purposes of a fungal metabolite screening program, it seems clear that rice would serve well as a standard culture medium. The data supporting the use of rice as an ideal medium may not be surprising to researchers who routinely culture fungi; this study validates the empirical knowledge of many mycologists and natural products researchers who use rice as a go-to culture substrate. While liquid media did not perform well in this study, they should not be discounted as a viable culture condition in other circumstances. Different microbes, such as those found in marine environments, may be far more suited to a liquid growing environment than those cultured here. However, new metabolites have been reported when marine fungi have been grown on rice (Shang et al., 2012), which supports the use of complex solid media as a standard culture condition.

\section{Additional file}

Additional file 1: UPLC-ELSD chromatograms of G142 in liquid media. The column was a BEH-C18, and the gradient increased linearly from 15:85 CH3CN:H2O to 100:0 over 10 min. Abbreviations of media are defined in the Methods section.

\section{Competing interests}

The authors declare that they have no competing interests.

\section{Acknowledgements}

The authors thank the City of Greensboro for permission to collect samples.

Received: 6 December 2013 Accepted: 11 December 2013

Published: 17 December 2013

\section{References}

Bills GF, Platas G, Fillola A, Jimenez MR, Collado J, Vicente F, Martin J, Gonzalez A, Bur-Zimmermann J, Tormo JR, Pelaez F (2008) Enhancement of antibiotic and secondary metabolite detection from filamentous fungi by growth on nutritional arrays. J Appl Microbiol 104:1644-1658 
Bills GF, Dombrowski AW, Goetz MA (2012) The "FERMEX" method for metabolite-enriched fungal extracts. In: Keller NP, Turner G (eds) Fungal Secondary Metabolism, vol 944. Humana Press, New York, pp 79-96

Bode HB, Bethe B, Hofs R, Zeeck A (2002) Big effects from small changes: Possible ways to explore nature's chemical diversity. ChemBioChem 3:619-627

El-Elimat T, Zhang X, Jarjoura D, Moy FJ, Orjala J, Kinghorn AD, Pearce CJ, Oberlies NH (2012) Chemical diversity of metabolites from fungi, cyanobacteria, and plants relative to FDA-approved anticancer agents. ACS Med Chem Lett 3:645-649

El-Elimat T, Figueroa M, Ehrmann BM, Cech NB, Pearce CJ, Oberlies NH (2013a) High-resolution MS, MS/MS, and UV database of fungal secondary metabolites as a dereplication protocol for bioactive natural products. J Nat Prod 76:1709-1716

El-Elimat T, Figueroa M, Raja HA, Adcock AF, Kroll DJ, Swanson SM, Wani MC, Pearce CJ, Oberlies NH (2013b) Waol A, trans-dihydrowaol A, and cisdihydrowaol A: Polyketide-derived $\gamma$-lactones from a Volutella species. Tetrahedron Lett 54:4300-4302

El-Elimat T, Figueroa M, Raja HA, Graf TN, Adcock AF, Kroll DJ, Day CS, Wani MC, Pearce CJ, Oberlies NH (2013C) Benzoquinones and terphenyl compounds as phosphodiesterase-4B inhibitors from a fungus of the order Chaetothyriales (MSX 47445). J Nat Prod 76:382-387

Ezekiel CN, Sulyok M, Warth B, Krska R (2012) Multi-microbial metabolites in fonio millet (acha) and sesame seeds in Plateau State, Nigeria. Eur Food Res Technol 235:285-293

Fiedurek J, Gromada A, Jamroz J (1996) Effect of medium components and metabolic inhibitors on $\beta$-galactosidase production and secretion by Penicillium notatum 1. J Basic Microb 36:27-32

Figueroa M, Raja H, Falkinham JO, Adcock AF, Kroll DJ, Wani MC, Pearce CJ, Oberlies NH (2013) Peptaibols, tetramic acid derivatives, isocoumarins, and sesquiterpenes from a Bionectria sp. (MSX 47401). J Nat Prod 76:1007-1015

Gloer JB (2007) Applications of fungal ecology in the search for new bioactive natural products. In: Wicklow DT, Soderstrom BE (eds) The Mycota, vol IV. Springer-Verlag, New York, pp 257-283

Hestbjerg H, Nielsen KF, Thrane U, Elmholt S (2002) Production of trichothecenes and other secondary metabolites by Fusarium culmorum and Fusarium equiseti on common laboratory media and a soil organic matter agar: an ecological interpretation. J Agr Food Chem 50:7593-7599

Keating G, Figgitt D (2003) Caspofungin: a review of its use in oesophagea candidiasis, invasive candidiasis and invasive aspergillosis. Drugs 63:2235-2263

Kimura Y, Katagiri K, Inoue T, Tamura S (1971) Isolation and biological activity of pestalotin, a gibberellin synergist from Pestalotia cryptomeriaecola. Agr Biol Chem 35:1313-1314

Kimura Y, Suzuki A, Tamura S, Mori K, Oda M, Matsui M (1977) Biological activity of pestalotins on the elongation growth of rice seedlings. Plant Cell Physiol 18:1177-1179

Kirihata M, Ohe M, Ichimoto I, Kimura Y (1996) Synthesis and biological activity of LL-P880 $y$ and its analogs. Biosci Biotechnol Biochem 60:677-679

Kirk PM, Cannon PF, Minter DW, Stalpers JA (2011) Dictionary of the Fungi, 10th edn. CABI Publishing, Wallingford

Kossuga MH, Romminger S, Xavier C, Milanetto MC, Do VMZ, Pimenta EF, Morais RP, De CE, Mizuno CM, Coradello LFC, Barroso VDM, Vacondio B, Javaroti DCD, Seleghim MHR, Cavalcanti BC, Pessoa C, Moraes MO, Lima BA, Goncalves R, Bonugli-Santos RC, Sette LD, Berlinck RGS (2012) Evaluating methods for the isolation of marine-derived fungal strains and production of bioactive secondary metabolites. Rev Bras Farmacogn 22:257-267

Miao L, Kwong TFN, Qian P-Y (2006) Effect of culture conditions on mycelial growth, antibacterial activity, and metabolite profiles of the marine-derived fungus Arthrinium c.f. saccharicola. Appl Microbiol Biotechnol 72:1063-1073

Mikusova P, Srobarova A, Sulyok M, Santini A (2013) Fusarium fungi and associated metabolites presence on grapes from Slovakia. Mycotoxin Res 29:97-102

Mohanty SS, Prakash S (2009) Effects of culture media on larvicidal property of secondary metabolites of mosquito pathogenic fungus Chrysosporium lobatum (Moniliales: Moniliaceae). Acta Trop 109:50-54

Paranagama PA, Wijeratne EMK, Gunatilaka AL (2007) Uncovering biosynthetic potential of plant-associated fungi: effect of culture conditions on metabolite production by Paraphaeosphaeria quadriseptata and Chaetomium chiversii. Nat Prod 70:1939-1945

Pu X, Qu X, Chen F, Bao J, Zhang G, Luo Y (2013) Camptothecin-producing endophytic fungus Trichoderma atroviride LY357: isolation, identification, and fermentation conditions optimization for camptothecin production. Appl Microbiol Biotechnol 97:9365-9375

Raja HA, Schmit JP, Shearer CA (2009) Latitudinal, habitat and substrate distribution patterns of freshwater ascomycetes in the Florida Peninsula. Biodivers Conserv 18:419-455

Scherlach K, Hertweck C (2006) Discovery of aspoquinolones A-D, prenylated quinoline-2-one alkaloids from Aspergillus nidulans, motivated by genome mining. Org Biomol Chem 4:3517-3520

Schoch CL, Seifert KA, Huhndorf S, Robert V, Spouge JL, Levesque CA, Chen W (2012) Nuclear ribosomal internal transcribed spacer (ITS) region as a universal DNA barcode marker for Fungi. Proc Natl Acad Sci USA 109:6241-6246

Schulz B, Wanke U, Draeger S, Aust HJ (1993) Endophytes from herbaceous plants and shrubs - effectiveness of surface sterilization methods. Mycol Res 97:1447-1450

Shang Z, Li X-M, Li C-S, Wang B-G (2012) Diverse secondary metabolites produced by marine-derived fungus Nigrospora sp. MA75 on various culture media. Chem Biodivers 9:1338-1348

Shearer CA, Langsam DM, Longcore JE (2004) Fungi in freshwater habitats. In: Mueller GM, Bills GF, Foster MS (eds) Measuring and monitoring biological diversity: standard methods for Fungi. Smithsonian Institution Press, Washington DC, pp 513-531

Soerensen JL, Nielsen KF, Sondergaard TE (2012) Redirection of pigment biosynthesis to isocoumarins in Fusarium. Fungal Genet Biol 49:613-618

Stone JK, Polishook JD, White JF Jr (2004) Endophytic Fungi. In: Foster M, Bills G (eds) Biodiversity of Fungi: Inventory and Monitoring Methods. Academic Press, Burlington, pp 241-270

Strader CR, Pearce CJ, Oberlies NH (2011) Fingolimod (FTY720): a recently approved multiple sclerosis drug based on a fungal secondary metabolite. J Nat Prod 74:900-907

Strunz GM, Heissner CJ, Kakushima M, Stillwell MA (1974) Metabolites of an unidentified fungus: a new 5,6-dihydro-2-pyrone related to pestalotin. Can J Chem 52:825-826

Xu P, Ding Z-Y, Qian Z, Zhao C-X, Zhang K-C (2008) Improved production of mycelial biomass and ganoderic acid by submerged culture of Ganoderma lucidum SB97 using complex media. Enzyme Microb Technol 42:325-331

doi:10.1186/2191-0855-3-71

Cite this article as: VanderMolen et al:: Evaluation of culture media for the production of secondary metabolites in a natural products screening program. AMB Express 2013 3:71.

\section{Submit your manuscript to a SpringerOpen ${ }^{\odot}$ journal and benefit from:}

- Convenient online submission

- Rigorous peer review

- Immediate publication on acceptance

- Open access: articles freely available online

- High visibility within the field

- Retaining the copyright to your article

Submit your next manuscript at $>$ springeropen.com 